\title{
Die familiäre Belastung der Tuberkulösen und ihre Beziehungen zu Infektion und Vererbung.
}

\author{
Von \\ Dr. med. W. Weimberg \\ in Stuttgart.
}

\section{Einleitung und Methode.}

Unter familiärer Belastung verstehe ich lediglich die reine Tatsache des Vorkommens weiterer Fälle einer Krankheit in der Familie eines davon Befallenen. Inwieweit sie im Sinne von Vererbung oder Wirkung gemeinsamer äusserer Schädlichkeiten, insbesondere Infektion $z u$ deuten ist, muss für jede einzelne Krankheit gesondert untersucht werden. Bei der Tuberkulose speziell ist bis jetzt noch nicht einmal entschieden, ob der Belastung überhaupt eine ätiologische Rolle zukommt. Mit dieser Frage und den Möglichkeiten ihrer Deutung hat sich die vorliegende Untersuchung beschäftigt, die ich hoffe, später durch reichlicheres Material ergänzen zu können.

Wenn man den Inhalt der soeben erschienenen zweiten Auflage von Cornets Werk, "Die Tuberkulose“, mit demjenigen der 1899 erschienenen ersten vergleicht, so wird man bei dem Kapitel Heredität keine wesentlichen Unterschiede der Darstellung und Beweisführung finden. Insbesondere sind neuere Arbeiten, welche ein Bestehen der Heredität beweisen sollen, so gut wie gar nicht im Text angeführt, wohl aber solche, welche gegen das Bestehen einer solchen zu sprechen scheinen, wie hauptsächlich die von Schwarzkopf und Reiche. Dabei verzichtet Cornet darauf, Fälle von Tuberkulose in zwei Generationen auf frühzeitige und lange latente Infektion der Kinder durch die Eltern zurückzuführen, da er eine lange Latenz der Tuberkulose überhaupt nicht als bewiesen anerkennt, vielmehr hält er es auch für falsch, in.jedem Fall, ohne Rücksicht auf die Länge des zwischen der Erkrankung bezw. dem Tod des einen und anderen Indi- 
viduums liegenden Zeitraumes, die Tuberkulose der Eltern als Ursache der Infektion der Kinder anzusehen; auch in solchen Fällen ist nach seiner Ansicht bei langem Intervall weit mehr an eine Infektion ausserhalb der Familie zu denken.

Es fragt sich nun, ob auf Grund einer solchen Einschränkung der Bedeutung latenter Infektion sich die Bedenken gegen das Bestehen hereditärer Finflüsse auf die Disposition zur Tuberkulose oder besser auf die Widerstandskraft des Organismus gegen die Infektion auf die Daner aufrecht erhaiten lassen. Alterdings sind die Gründe, welche Cornet für seine herbe Kritik der bisherigen Versuche, das Bestehen einer Vererbung zu beweisen, ins Feld geführt hat, durchaus anzuerkennen. Insbesondere muss die bisherige zugunsten der Heredität angeführte Statistik als fast durchweg auf unrichtiger oder ungenauer Fragestellung beruhend bezeichnet werden. Ich habe daher auch davon Abstand genommen mich mit ihr hier nochmals im einzelnen auseinanderzusetzen. Nur die Arbeit von Westergaard entspricht den Anforderungen der statistischen Methodenlehre, beruht aber auf den unrollständigen Angaben einer Lebensversicherungsgesellschaft, deren Wert ich frïher gekennzeichnet habe. Indessen kann eine Lehre damit, dass sie ais ungenügend begrïndet nachgewiesen wird, nicht als für alle Zeit abgetan betrachtet werden, solange nicht direkte Beweise ihrer absoluten Unrichtigkeit vorliegen. Das beste Beispiel dafür ist die Geschichte der Lehre der Infektiosität der Tuberkulose selbst, die ja vielleicht ebenso alt oder älter ist als die Lehre von der Vererbung, ohne dass die dafür beigebrachten Beweise vor dem Zeitalter der Entdeckung Robert $\mathrm{Kochs}$ als genügend betrachtet werden konnten. Das hat an der Richtigkeit der Lehre von der Infektiosität der Tuberkulose nichts geändert. Dass aber die Infektiosität das Bestehen hereditärer Einflüsse nicht absolut ausschliesse, hat $\mathrm{Koch}$ selbst in seiner ersten Veröffentlichung noch zugegeben.

Es ist also in erster Linie zu untersuchen, ob die Beweise gegen das Bestehen hereditärer Einflüsse bei der Entstehung der Tuberkulose als erdrückend angesehen werden müssen.

Dies kann nun vorläufig nicht zugegeben werden.

Cornet will nur solche Statistiken als beweisend anerkennen, welche von Fällen ausgeben, in denen eine Infektionsmöglichkeit durch die Familie $n$ icht bestand. Die Arbeit von $S c h w a r z k$ p $f$ scheint nun zunächst dafür zu sprechen, dass in solchen Fällen ein Einfluss der Heredität ausgeschlossen ist. Schwarzkopf fand nämlich eine vermehrte Häutigkeit der Tuberkulose bei den Eltern Tuberkulöser im Vorgleich mit denen Nichttuberkulöser nur in denjenigen Fällen, 
in denen auch die Möglichkeit einer Infektion in der Familie bestanden hatte.

Indessen kann die Untersuchung von $\mathrm{S} \mathrm{chwarzk}$ opf keineswegs als den Anforderungen der wissenschaftlichen Statistik durchaus entsprechend bezeichnet werden. Schwarzkopf gibt lediglich an, dass die von ihm untersuchten Frauen alle über 13 Jahre alt waren, aber wie sich im einzelnen der Altersaufbau der Tuberkulösen und Nichttuberkulösen, der Fälle mit und ohne Gelegenheit zur Infektion in der Familie, gestaltete, darüber ist nichts mitgeteilt. Und doch beruht die ganze Beweiskraft der Arbeit Schwa r zk op fs vom Gesichtspunkt des Statistikers lediglich auf der stillschweigenden Voraussetzung, es hätten die von ihm verglichenen Gruppen denselben Altersaufbau oder mindestens dasselbe Durchschnittsalter gehabt. Wenn dies aber nicht der Fall war, so kann die fehlende Mehrbelastung der nicht einer Infektionsgelegenheit in der Familie ausgesetzt gewesenen Tuberkulösen auch lediglich auf dem Umstande beruhen, dass diese durchschnittlich jünger waren als die mit ihnen verglichenen Nichttuberkulösen. Denn es leuchtet wohl ohne weiteres ein, dass ein altes Individuum eine vollständigere Übersicht über die Todesursachen seiner Eltern und Geschwister zu geben vermag als ein jugendliches, denn die Dauer der Beobachtung der Eltern und Geschwister wächst mit dem eigenen Alter eines Individuums. Auch unter sonst gleichen Umständen werden vierzigjährige Individuen mehr Todesfälle an Tuberkulose in ihrer Familie aufzählen können als zwanzigjährige. Eine verschiedene Mischung der Altersklassen bei zwei zu vergleichenden Gruppen kann daher Unterschiede sowohl vortäuschen als verwischen. Dieser Fundamentalsatz der wissenschaftlichen Statistik macht auch vor den Schranken der Hereditätsforschung nicht Halt; diese muss sich der gleichen Exaktheit befleissigen wie die übrige statistische Forschung; der Einfluss des Alters muss notwendig ausgeschaltet sein, ehe irgend eine Arbeit als einwandfrei bezeichnet werden kann, von welcher Seite auch sie kommen mag.

Dieselben Bedenken gelten auch gegenüber der Arbeit von K u thy.

Ebensowenig wie das Ergebnis der Arbeit von Schwarzkopf können die Resultate der Arbeiten von Reiche als ausschlaggebend bezeichnet werden.

Reiche fand bei den seitens ihrer Eltern belasteten Tuberkulösen eine sogar etwas geringere Kindersterblichkeit als bei den nichtbelasteten Tuberkulösen. Dabei versteht er unter Kindersterblichkeit die Sterblichkeit innerhalb der ersten zehn Lebensjahre. Daraus schliesst er, dass ein Einfluss der Belastung durch die Eltern mit Tuberkulose keinen Einfluss auf die Vitalität der Kinder ausübe. 
Dagegen lässt sich nun einwenden, dass das Material von $R$ e iche zur Entscheidung der Frage nicht ausreicht, da es sich lediglich um die Erfahrungen bei den Geschwistern Erwachsener handelt. Bei solcher Abgrenzung sind diejenigen Familien ausgeschlossen, in welchen keines der Kinder über das Alter der Pubertät hinaus am Leben blieb, und es muss als möglich hingestellt werden, dass deren Zahl unter den Familien Tuberkulöser häufiger ist als unter denen Nichttuberkulöser. Die Berechtigung dieses Einwandes werde ich im Laufe dieser Untersuchung an eigenem Materiale nachweisen.

Weiterhin hat Reiche bei den Personen, bei welchen seitens der Hamburger Versicherungsanstalt ein Heilverfahren wegen Tuberkulose eingeleitet wurde, gefunden, dass bei den hereditär Belasteten mindestens ebenso günstige Ergebnisse erzielt wurden wie bei den Nichtbelasteten. Auch dieses Ergebnis scheint gegen eine ungünstige Wirkung elterlicher Belastung zu sprechen. Allein ehe dieses Ergebnis als im Sinne des Statistikers einwandfrei angesehen werden kann, müsste doch eine Anzahl ron Vorbedingungen erfüllt sein. Es müsste nachgewiesen sein, dass weder die Versicherungsanstalt selbst beziehungsweise die ein Heilverfahren beantragenden Ärzte bei familiär belasteten Individuen eine schärfere Auswahl in der Art der Fälle walten liessen, dass ferner die zum Heilverfahren Zugelassenen nach Lebensalter, Ausdehnung und Dauer der Krankheit in demselben Zahlenverhältnis zusammengesetzt waren wie die Nichtbelasteten. Nur dann hätte das Ergebnis des gleichen Heilerfolges bei beiden Gruppen der Erwartung entsprochen. Hat jedoch ein Teil der familiär Belasteten aus Furcht vor dem Schicksal der Eltern zeitiger ein Heilverfahren beantragt und erhalten als die Nichtbelasteten, so war a priori ein besserer Erfolg bei den Belasteten zu erwarten als bei den Nichtbelasteten; die tatsächliche Gleichheit des Erfolges würde dann doch ein für die Belasteten ungünstiges Ergebnis darstellen. Über alle diese Fragen verlautet bei Reiche nichts, wir wissen nicht, ob die von ihm verglichenen Belasteten und Nichtbelasteten statistisch vergleichbar waren, und damit ist der Wert seiner Arbeit ebenso wie die zu ähnlichen Ergebnissen gelangende Arbeit von Turban darauf reduziert, dass sie zu neuen, auch die erwähnten Fragen genauer berücksichtigenden Arbeiten Veranlassung geben kann.

Cornet weist endlich auf die günstigen Sterblichkeitsverhältnisse der familiär Belasteten in Waisenhäusern hin. Dagegen ist nur einzuwenden, dass nicht feststeht, ob und inwieweit die Waisenhäuser eine Auslese der gesünderen Individuen treffen und dass in dem Alter, während dessen die Kinder im Waisenhaus bleiben, die Sterblichkeit an Tuberkulose ibren tiefsten Stand erreicht. Zur Gewinnung ein- 
wandfreier Zahlen wären also sehr umfassende Beobachtungen nötig, wie sie vorläufig nicht vorliegen.

Nur eine Beobachtung scheint direkt gegen die Vererbung zu sprechen, das ist der stärkere Einfluss der tuberkulösen Mutter auf die Entstehung der Tuberkulose bei den Kindern. Indessen ist diese Frage noch keineswegs endgültig erledigt und lässt auch eine Erklärung $z u$, welche den Einfluss erblicher Momente keineswegs ausschliesst.

Vom Standpunkt des Statistikers erscheint eine Vererbung bei der Tuberkulose derzeit weder erwiesen noch widerlegt. Das Urteil über diese Frage kann daher nur von dem Stand der allgemeinen Auffassung der Ätiologie der Tuberkulose und von dem Ergebnis weiterer Untersuchungen abhängen.

Es muss nun zugegeben werden, dass die derzeitige Auffassung der Ätiologie der Tuberkulose der Annahme hereditärer Einflüsse von vornherein nicht sehr günstig erscheint. In erster Linie hat jede Untersuchung, die auf wissenschaftliche Beachtung Anspruch machen will, die Tatsache des infektiösen Charakters der Tuberkulose anzuerkennen und sich mit ihr in der Weise auseinanderzusetzen, dass festzustellen ist, wie gross der Einfluss der Belastung im Vergleich mit dem der Infektion ist. Bedenkt man nun, wie schwer es ist, allein für die Frage der Infektion, soweit es sich nicht um das Tierexperiment, sondern um Menschen handelt, zuverlässige statistische Anhaltspunkte zu gewinnen, so ergibt sich schon daraus, mit welchen Schwierigkeiten der statistische Nachweis erblicher Einflüsse zu kämpfen haben muss. In gewissem Sinne hat allerdings die Erkenntnis des infektiösen Charakters der Tuberkulose einen Fortschritt für das Problem der Vererbung gebracht, indem sie die Veranlassung wurde, alle unklaren Vorstellungen, welche bisher mit dem Begriff der Vererbung bei der Tuberkulose verbunden waren, abzustreifen und das Problem vom hiologischen Standpunkt genauer zu definieren. Die biologische Auffassung, als deren Hauptvertreter Weismann anzusehen ist, kennt keine Vererbung erworbener Eigenschaften und keine Übertragung von Krankheiten durch das Keimplasma, sondern nur die Übertragung von Anlagen, welche die Widerstandskraft beeinflussen. Streng davon zu trennen sind germinative und plazentare Infektion während des Fötallebens. Auch die Einwirkung von Toxinen tuberkulöser Eltern auf den Samen oder das Ei fällt streng genommen nicht unter den Begriff der Vererbung.

Für die statistische Fragestellung können diese Einflüsse allerdings nur schwer auseinandergehalten werden. Der Einfluss plazentarer Übertragung kainn allerdings unschwer ausgeschaltet werden, wenn 
man sich auf die Untersuchung der Vererbung durch den Vater beschränkt. In diesem Falle ist auch die Wirkung germinativer Infektion wenn auch nicht theoretisch unmöglich, so doch praktisch so gut wie ausgeschlossen und es kommt neben der Vererbung nur die Infektion nach der Geburt als ätiologisches Moment ausschliesslich oder teilweise in Frage.

Auch der Einfluss der Infektion durch den tuberkulösen Vater liesse sich ausschalten bei Beschränkung der Untersuchung auf solche Kinder, die erst nach dem Tode des tuberkulösen Vaters geboren wurden. Allein das ist bei der Seltenbeit des Materials eine Aufgabe, deren Lösung von langer Hand vorbereitet werden muss.

Erschwert wird weiterhin die Untersuchung durch die Erkenntnis des exquisit sozialen Charakters der Tuberkulose, den auch meine bisherigen eigenen Untersuchungen wieder bestätigt haben. Der Einfluss der sozialen Verhältnisse macht es an und für sich schon zu einem Postulat, dass bei Tuberkulösen auch in deren übriger Familie häufiger Tuberkulose gefunden werden muss als bei Nichttuberkulösen, selbst wenn alle übrigen Umstände gleich sind.

Der Versuch Cornets, mit Hilfe der ungenauen Statistik von Kuthy nachzuweisen, dass die Tuberkulösen nicht stärker familiär belastet sind als die Nichttuberkulösen, hat von diesem Gesichtspunkt etwas Planloses und beweisen wie leicht auch die Gegner der Dispositionslehre die Unbefangenheit verlieren. Das Eine geht aus der Erkenntnis des sozialen Charakters der Tuberkulose hervor, dass der Nachweis stärkerer familiärer Belastung allein nicht genügt für den Nachweis hereditärer Momente, sondern dazu muss mindestens eine Voraussetzung erfüllt sein, dass nämlich der Einfluss sozialer Momente ausgeschlossen ist. Dies bedeutet eine weitere gewaltige Erschwerung der Untersuchung. Von besonders ungünstiger Wirkung für die Lehre von der Heredität bei der Tuberkulose war weiterhin das Erscheinen der Arbeiten von Schlenker, Nägeli und Burkhart, welche fast bei jedem erwachsenen Toten Tuberkulose fanden, und der Theorie Behrings, welcher diese Ergebnisse im Sinne einer sehr frühzeitigen Durchseuchung der Menschheit mit Tuberkulose schon im Säuglingsalter und frühen Kindesalter erklärte; für die Erkrankungen der Erwachsenen eine lange Latenz annahm und ungünstige soziale und berufliche Verhältnisse lediglich als auslösendes Moment betrachtete. Diese Lehre war weit mehr als die berechtigte Kritik ihrer bisherigen Gegner geeignet, der Annahme hereditärer Einflüsse bei der Tuberkulose den Boden zu entziehen und wenn sie sich endgiiltig als richtig erweist, kann von einem Versuch des Nachweises solcher Eintlüsse kaum mehr 
die Rede sein. Zunächst erfreut sich jedenfalls diese Lehre wachsender Anerkennung.

Gegen die Verallgemeinerung der Sektionsergebnisse aus Spitälern sind allerdings berechtigte Einwände erhoben worden. In der der Tat stellte eine Übertragung dieser Ergebnisse auf die Lebenden einen Rückfall in jene als trügerisch erkannte Anschauungsweise dar, welche lediglich von den Gestorbenen ausgehend das Alter von 15-30 Jahren als das am stärksten der Infektion exponierte ansah, weil in ihm die Tuberkulose sowohl unter allen Gestorbenen wie unter denen gleichen Alters am häufigsten auftritt. Man ist sicher berechtigt bei den Lebenden einen geringeren Prozentsatz Tuberkulöser und tuberkulös Gewesener anzunehmen als bei den Toten. Das geht aus den Untersuchungen über die Häufigkeit positiver Tuberkulinreaktion bei Nichttuberkulösen zur Genüge hervor, und die Untersuchungen an den Leichen diphtheritischer, masern- und scharlachkranker Kinder stellten sicher das ungeeigenste Material zur Feststellung der Häufigkeit tuberkulöser Prozesse bei Lebenden dar, da die Tuberkulose den Verlauf dieser Krankheiten sowohl besonders ungünstig beeinflusst, als auch durch sie leicht wieder florid gemacht wird. Man kann daher mit Bestimmtheit annehmen, dass im Kindesalter die Tuberkulose bei Lebenden seltener ist als bei den an diesen Infektionskrankheiten Gestorbenen. Eine definitive Widerlegung der Behringschen Theorie darf man aber in der Reduktion der Anschauungen, welche sich auf die grosse Häufigkeit latenter Tuberkulose bei Spitalsektionen gründete, auf ihr berechtigtes Mass nicht erblicken, sondern lediglich eine vielleicht doch nicht sehr bedeutende Einschränkung, die es immer noch erlauben könnte, viele Fälle von Tuberkulose Erwachsener auf Infektion im frühen Kindesalter zurückzuführen. Auch der Einwand, dass die tuberkulöse Infektion bei Kindern im 1.-2. Lebensjahre fast stets tödlich verläuft, ist nicht absolut stichhaltig, dem kann entgegengehalten werden, dass diese Auffassung lediglich auf klinischen Erfahrungen berubt und die günstigeren Fälle eben nicht zur klinischen Beobachtung kommen. Einen ähnlichen Einwand musste ich den übertriebenen Vorstellungen ron dem Einfluss der Schwangerschaft auf Entstehung und Verlauf der Tuberkulose gegenüber auf Grund statistischer Ergebnisse machen. Immerhin muss man zugeben, dass die Entstehung aller Tuberkulosen im frühen Kindesalter eine derartige Häufigkeit vorläufiger Heilungen voraussetzen würde, wie man sie auch in der bestgeleiteten Lungenheilanstalt bei Erwachsenen leider nicht feststellen kann und dass die Seltenheit geheilter Tuberkulose bei Sektionen in diesem Alter entschieden gegen die Richtigkeit dieser Lehre spricht. 
Die Tatsache, dass die Tuberkulosesterblichkeit im ersten Lebensjahre höher ist als in jeder folgenden Altersklasse der Kinder und Erwachsenen, beweist noch nicht ohne weiteres, dass in ihm die meisten Infektionen vorkommen, sondern vielleicht nur, dass sie tatsächlich in diesem Alter am häufigsten letal ablaufen. Die relative Zahl der Infektionen kann also trotzdem in anderen Altersklassen, speziell bei Erwachsenen, wesentlich höher sein als im Kindesalter.

Die Theorie Behrings geht von der Einheit des Tuberkelbazillus aus. So lange die Unterscheidung mehrerer Typen nicht als abgetan gelten kann, scheint ein Widerspruch darin zu liegen, dass die dem Säugling verabreichte Kuhmilch als eine wesentliche Ursache der Tuberkulose Erwachsener betrachtet wird, in deren Sputum sich der Typus humanus findet.

Jedenfalls bestehen über das Vorkommen einer langdauernden Latenz der Infektion erheblicheVerschiedenheitender Auffassung zwischen Cornet und Behring. Daher besteht immer noch eine gewisse Berechtigung, die Frage nach der Deutung des Einflusses familiärer Belastung aufs neue in Angriff zu nehmen. Dazu kommt, dass die Frage nach der Grösse dieses Einflusses insofern auch praktisch nicht ohne Bedeutung ist als namentlich bei den Lebensversicherungsgesellschaften die Tuberkulose der Eltern einen Grund bildet die Gesundheitsverhältnisse eines Antragstellers besonders streng zu prüfen. Daher dürfte eine nach den Grundsätzen der wissenschaftlichen Statistik durchgeführte Untersuchung, zu welcher bereits vor dem Auftreten der Theorie Behrings der Plan vorlag und die Sammlung des Materials begonnen hatte, immerhin auf Beachtung rechnen.

Meine Untersuchung zerfällt in zwei Teile. Ich habe sowohl den Einfluss der tuberkulösen Väter und Mütter auf die Sterblichkeit der Kinder überhaupt und an Tuberkulose bis zum zwanzigsten Lebensjahre untersucht.

Weiterhin habe ich auch bei Erwachsenen die Häufigkeit der Belastung durch Eltern und Geschwister bei Ausschluss des Einflusses von Alter und sozialen Verhältnissen für Tuberkulöse und Nichttuberkulöse festgestellt.

Als Material dienten mir Zählkarten, welche auf Grund der Stuttgarter Totenscheine der Jahre 1873-1902 hergestellt wurden (siehe W ein berg 2). Die Methode der Untersuchung musste in erster Linie auf die Herstellung der Möglichkeit eines Vergleiches gerichtet sein. An dem Mangel eines solchen Vergleiches sind die meisten bisherigen Arbeiten über pathologische Heredität auch auf anderen Gebieten gescheitert und dieser Mangel ist auch die Hauptstütze der scharfen Kritik, welche Cornet an den bisherigen Arbeiten über Heredität bei Tuberkulose geübt hat. 
Auch das wertvolle von $\mathrm{Riffel}$ beigebrachte Material leidet unter dem Mangel einer Verarbeitung nach statistischen Grundsätzen, die übrigens noch nachgeholt werden kann. Die von mir bei der Untersuchung der Erwachsenen verfolgte Methode habe ich in ihrer theoretischen Bedeutung bereits in meiner Arbeit über pathologische Vererbung und genealogische Statistik im Jahre 1903 beschrieben und sie auch auf die Frage der Vererbung des Krebses und seiner fraglichen hereditären Beziehungen zur Tuberkulose angewandt. Sie besteht im Vergleich der familiären Belastung verheirateter Tuberkulöser mit derjenigen ihrer Ehegatten und kann auch auf Erfahrungen in der hausärztlichen Praxis ohne weiteres angewandt werden. Diese Methode hat den Vorteil, dass sie der von Cornet gestellten Forderung, wonach sich die Untersuchung der Heredität nur auf Personen erstrecken soll, die keine Ansteckungsgelegenheit in der Familie hatten, insofern zutrifft, als in den meisten Fällen die Heirat der Belasteten erst längere Zeit nach dem Tode der tuberkulösen Eltern stattfand. Hingegen schliesst sie die tuberkulösen Ledigen von der Untersuchung aus. Dies hat den Nachteil, dass es sich bei der Untersuchung um Erwachsene von durchschnittlich ziemlich gesetztem Alter handelt, bei denen man a priori einen verhältnismässig geringen Einfluss der Vererbung auch dann erwarten wird, wenn eine solche überhaupt besteht. Von um so grösserer Bedeutung für die Gesamtheit der Erwachsenen dürfte ein trotzdem gefundenes positives Ergebnis sein. Man kann der Untersuchung jedenfalls nicht den Vorwurf machen, dass sie sich ihre Vorbedingungen zu günstig ausgewählt $\cdot$ habe.

Die vorliegende Arbeit könnte ein weit grösseres Material umfassen, wenn nicht die dafür gesammelten Mittel erschöpft wären und ich selbst durch lange Erkrankung verhindert worden wäre, weitere eigene Opfer dafür zu bringen. Nach meiner Rekonvaleszenz sehe ich mich veranlasst, die Arbeit wenigstens vorläufig abzuschliessen und ihre Fortsetzung von dem Zugang weiterer Mittel abhängig zu machen. Um eine weitere Verwendung und Ergänzung des Materials zu ermöglichen, habe ich das gesamte Urmaterial meiner Untersuchungen über Krebs und Tuberkulose in Stuttgart auf der dortigen Landesbibliothek deponiert, wo es später auch anderen Arbeitern auf diesem Gebiete von Nutzen sein kann.

\section{Der Einfluss tuberkulöser Väter und Mütter auf die Sterblich- keit ihrer Kinder bis zum zwanzigsten Lebensjahre.}

Bei denjenigen verheirateten Tuberkulösen, welche 1873-1882 starben und deren Ehe in den Stuttgarter Familienregistern eingetragen war (dies sind ca. $95 \%$ aller Fälle), wurde aus diesen Registern Geburt und Tod des letzten ehelichen Kindes, ausserdem die 
Todesursache aus Kirchenbüchern, bei den Todesfällen nach 1850 aus den Leichenscheinen ermittelt, soweit dies ohne Anfragen nach auswärts möglich war ${ }^{1}$ ). (Über die Einrichtung der württembergischen Familienregister siehe Weinberg und Gastpar, Die bösartigen Neubildungen in Stuttgart 1873-1902, Zeitschrift für Krebsforschung Bd. II., S. 197).

Untersuchungen über das Schicksal sämtlicher Kinder dieser Familien müssen einer späteren Zeit vorbehalten werden ${ }^{2}$ ).

Da ein Teil der Kinder mit den überlebenden Ehegatten der Tuberkulösen nach auswärts gezogen ist, fallen die berechneten Verhältniszahlen notwendig zu klein aus, doch ist der Einfluss dieses Fehlers nicht von grosser Bedeutung für das Ergebnis, er liesse sich auch, da die Zeit des Wegzuges der Eltern bekannt ist, ganz ausschalten; auch dies muss auf später verschoben werden. Die Untersuchung umfasst die Kinder von 717 sicher tuberkulösen Vätern und 434 sicher tuberkulösen Müttern. Diejenigen, welche zwei tuberkulöse Eltern hatten. sind doppelt gezählt. Das Geschlecht der Kinder ist nicht ausgeschieden, der Unterschied in der Verteilung der Geschlechtes dürfte wohl nicht wesentlich in Betracht kommen.

Es starben bis Ende 1902, soweit ermittelt, Kinde1

$$
\text { tuberkulöser Väter tuberkulöser Mütter }
$$

vor der Geburt 36

im 1. Lebensjahr

im 2.--20. Lebensjahr

77

62 ,

darunter im 2.-20. Lebensjahr an Tuberkulose

ohne unsichere Fälle

mit unsicheren Fällen

25.

Die Häufigkeit der Tuberkulose im ersten Lebensjahre wurde mit Rücksicht auf die Unvollkommenheit der Todesursachenstatistik dieses Alters nicht festgestellt. Als unsichere Fälle sind gezählt: Lungenleiden, Abzehrung, Brustleiden.

Die Totgeburtenziffer betrug bei den Kindern

$$
\begin{aligned}
& \text { tuberkulöser Väter } \mathbf{5 , 0} \% \\
& \text { Mütter } 6,2, \% \text {. }
\end{aligned}
$$

Beide Ziffern sind als hoch zu bezeichnen, teilweise entspricht dies dem ungünstigen sozialen Durchschnittsniveau der Eltern, teil-

1) Diese Erbebungen wurden auch für 1883-1902 gemacht, sind aber noch nicht verarbeitet.

2) Eine solche hat bereits Kirchner auf Grund des von Riffel gesammelten Materials vorgenommen, ohne jedoch den Einfluss des Alters zu berücksichtigen. Dasselbe gilt für die Untersuchungen von Jakob und Pannwitz. 
weise der Tatsache, dass Letztgeborene eine höhere Totgeburtenziffer haben. Der Einfluss der Mutter auf die Vitalität der Kinder erscheint stärker als der des Vaters.

Die Sterblichkeit im ersten Lebensjahre betrug bei den

Kindern tuberkulöser Väter $31 \%$

$$
\text { Mütter } 38 \% \text { der }
$$

Lebendgeborenen. Auch hier erscheint der Einfluss der tuberkulösen Mutter stärker als der des tuberkulösen Vaters. Die durchschnittliche eheliche Kindersterblichkeit betrug in Stuttgart während der in Betracht kommenden Zeiträume etwa 22-25\%. Nimmt man an, dass die Letztgeborenen mit ihrer Kindersterblichkeit ebenso hoch über dem Durchschnitt stehen, wie die Erstgeborenen unter ihm, so müsste man als erwartungsmässige Ziffer auf Grund der Erfahrungen von Geissler ein Plus von ca. 2\%, also 24-27\% annehmen. Dazu käme mit Rücksicht auf das ungünstige soziale Durchschnittsniveau der Kinder Tuberkulöser noch ein Zuschlag von 10\% obiger Werte, also eine Vergleichszahl von der Höhe 26,4-29,7\%. Danach würde sich auch die Sterblichkeit der Kinder tuberkulöser Väter im ersten Lebensjahre noch etwas höher beziffern als ihrem sozialen Durchschnitt entspricht. Bedenkt man, dass die Tuberkulose bei der Gesamtbevölkerung, auch unter Zugrundelegung der Sektionsergebnisse von Schlossmann höchstens $7 \%$ der Todesfälle im ersten Lebensjahre ausmacht, so kann dieser Unterschied nicht als gering angesehen werden, für die Kinder tuberkulöser Frauen ist er noch weit erheblicher.

Von den das erste Lebensjahr überlebenden Kindern starben, soweit bekannt, bis Ende 1902

$$
\begin{array}{ccc}
\text { bei Tuberkulose des Vaters } & 16,6 \% \\
& & \text { der Mutter } 24,5 \%
\end{array}
$$

darunter an Tuberkulose einschliesslich unsichere Fälle 4,5\% bezw. $9,9 \%$.

Auch hier erscheint der Einfluss tuberkulöser Mütter wieder bedeutend erheblicher als derjenige tuberkulöser Väter.

Das 20. Lebensjahr vollendeten lebend von den

Kindern tuberkulöser Väter $57,7 \%$

Mütter $47,0 \%$

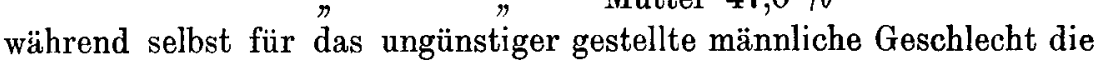
deutsche Sterbetafel am Ende des 20. Lebensjahres noch 59\%, für das Nittel beider Geschlechte aber etwa $61 \%$ ergibt $^{1}$ ). Das Ergebnis ist also

1) Dabei ist die deutsche Sterbetafel noch zu ungünstig, weil sie auf den Erfahrungen von 1871-1881 beruht, während die Beobachtung der Kinder der Tuberkulösen grossenteils auf eine spätere Zeit fällt. 
auch für die Kinder tuberkulöser Väter ungünstig, namentlich weil die Unkenntnis eines Teils der auswärtigen Todesfälle die Lebenserwartung der Kinder tuberkulöser Eltern zu hoch erscheinen lässt, aber lange nicht in dem Mass wie für die Kinder tuberkulöser Mütter; diese sind unter den Erwachsenen wesentlich weniger häufig als unter den Lebendgeborenen.

Noch weit erheblicher erscheint der Einfluss der Tuberkulose der Eltern, wenn man lediglich die in deren letzten sieben Lebensjahren geborenen Kinder in Betracht zieht. Dies waren 435 Kinder tuberkulöser Väter, und 314 Kinder tuberkulöser Mütter.

Von diesen starben Kinder tuberkulöser

$\begin{array}{ccc} & \text { Väter } & \text { Mütter } \\ \text { vor der Geburt } & 21 & 21 \\ \text { im 1. Lebensjabre } & 148 & 129 \\ \text { im 2.-20. Lebensjahre } & 59 & 45 \\ \text { unter letzteren an Tuberkulose } & 8 & 15 \\ \text { Totgeburtenziffer betrug bei den Kindern } & \\ \text { tuberkulöser Väter } 6,5 \% & \\ " \text { Mütter } 6,7 \% & \end{array}$

war also wesentlich höher als im Durchschnitt aller Kinder tuberkulöser Eltern.

Im ersten Lebensjahre starben von den lebendgeborenen Kindern tuberkulöser Väter $36,9 \%$

und im 2.-20. Lebensjahre Mütter $44,1 \%$

$$
\begin{array}{ccc}
\text { von den Kindern tuberkulöser Väter } & 23,3 \% \\
, ", \quad & \text { Mütter } 27,4 \%
\end{array}
$$

der über ein Jahr alt gewordenen. Der Unterschied in dem Einfluss beider Eltern erscheint hier eher kleiner wie bei Betrachtung aller Fälle. Wenn dieses Ergebnis sich auch bei grösserem Material bestätigen sollte, so kann es nur dadurch erklärt werden, dass der Einfluss der tuberkulösen Mutter sich infolge des intimeren Zusammenlebens mit den Kindern auch dann stark geltend macht, wenn sie verhältnismässig längere Zeit nach der Geburt eines Kindes an Tuberkulose stirbt, beziehungsweise an offener Tuberkulose leidet. Dies wird dadurch bestätigt, dass von den Lebendgeborenen derjenigen Eltern, welche mehr als sieben Jahre nach dem Tode ihrer letztgeborenen Kinder starben, bei Tuberkulose

$\begin{array}{ccc} & \text { des Vaters } & \text { der Mutter } \\ \text { im ersten Lebensjahre starben } & 64=23 \% & 25=22 \% \\ \text { von den über ein Jahr alt gewordenen } & \\ \text { bis zum 20. Lebensjahre } 18=8,3 \% & 17=19 \%\end{array}$


Die Tuberkulose der Eltern erscheint demnach besonders in demjenigen Alter gefährlich für die Kinder, in dem sie als manifest anzuseben ist.

Von den in den letzten sieben Lebensjahren der tuberkulösen Eltern lebendgeborenen Kindern erreichten bei

Tuberkulose des Vaters nur $48,4 \%$
,,$\quad$ der Mutter $40,6 \%$

das 20. Lebensjahr. Diese Fälle sind also unter den Erwachsenen verhäItnismässig schwächer vertreten als diejenigen mit spätem Tod der Eltern, dies muss in dem Sinn wirken, dass die Wirkung der familiären Belastung Erwachsener zu schwach erscheint.

Mit der Gesamtbevölkerung lassen sich die Zahlen bei Unterscheidung nach der Zeit des Todes der Eltern nicht mehr vergleichen, weil diese Zeit auch bei anderen Todesursachen einen Einfluss auf die Sterblichkeit der Kinder haben kann.

Die Untersuchung konnte nur bis zum 20. Lebensjahre ausgedehnt werden, da die letztgeborenen Kinder der Tuberkulösen, welche 1873-1882 starben, Ende 1902 zu einem grossen Teil erst im 3. Dezennium des Lebens standen. Es wird später möglich sein, ihr weiteres Schicksal und damit die Wirkung der Tuberkulose der Eltern auf das Schicksal der erwachsenen Kinder festzustellen.

Ganz besonders ungünstig ist es, wenn die Kinder im letzten Lebensjahr der Eltern geboren werden. Ich habe die Untersuchung hierüber auf alle in der Zeit von 1873-1902 geborenen Kinder Tuberkulöser ausgedehnt. Von 366 Kindern tuberkulöser Väter, die innerhalb eines Jahres nach der Geburt ihrer letzten Kinder starben, waren totgeboren $16=4,4 \%$, von den 350 Lebendgeborenen starben im 1. Lebensjahr $165=50 \%$, also gerade die Hälfte; von den entsprechenden 343 Kindern tuberkulöser Mütter waren totgeboren $22=6,4 \%$, von den Überlebenden starben $217=68 \%$ oder über zwei Drittel im 1. Lebensjahr und überlebte, soweit nachweisbar, keines das 21. Lebensjahr. Von den 57 im letzten Lebensmonat ihrer tuberkulösen Mütter geborenen Kinder waren totgeboren $10=17,5 \%$ und es starben von den Lebendgeborenen $37=79 \%$ im 1. Lebensjahr.

Von 103 Kindern, die erst innerhalb 9 Monaten nach dem Tode ihrer tuberkulösen Väter geboren wurden, waren totgeboren $4=3,9 \%$, von den 99 Lebendgeborenen starben im 1 . Lebensjahr $36=36 \%$. Diese Ziffer ist höher als die Sterbeziffer der unehelich geborenen Kinder in Württemberg, welche 1873-1902 $33 \%$ betrug. In einem Teil dieser Fälle wird man geneigt sein, einen illegitimen aber gesunden Vater als tatsächlichen Erzeuger anzusehen; wenn dies be- 
rechtigt ist, so würde die hole Sterbeziffer der Nachgeborenen in noch ungünstigerem Lichte erscheinen.

Aus diesen Ergebnissen geht hervor, dass die Kinder tuberkulöser Eltern um so weniger Wahrscheinlichkeit haben das Alter der Erwachsenen zu erreichen, je näher ihre Geburt dem Tode ihrer Eltern liegt und je mehr Gelegenheit sie hatten in frühem Kindesalter mit offener Tuberkulose der Eltern in Berührung zu kommen. Dass aber die ganze Übersterblichkeit der Kinder tuberkulöser Eltern lediglich auf Rechnung der Tuberkulose komme, lässt sich auf Grund dieser Daten nicht mit Bestimmtheit behaupten. Dazu müsste man die Todesursachen oder richtiger den Leichenbefund aller solchen Kinder kennen. In der Zeit nach dem ersten Lebensjahr kommt ein Teil der Übersterblichkeit der Kinder tuberkulöser Eltern jedenfalls auf Rechnung von solchen Todesfällen, bei denen ein Vorhandensein tuberkulöser Prozesse nicht intra vitam konstatiert wurde. Für die höhere Sterblichkeit der Kinder tuberkulöser Mütter kommt auch die grössere Häufigkeit von Frühgeburten, die nach Schmorl nicht geringe Häufigkeit plazentarer Infektion, weiterhin vor allem aber die geringere Aussicht auf genügende und zweckmässige Ernährung des Säuglings, als Ursache in Betracht. Der tuberkulösen Mutter pflegt ja das Stillen rerboten zu werden, in vorgeschrittenen Stadien der Tuberkulose verbietet es sich ohnehin von selbst. Aber auch die Krankheit des Vaters wirkt vermöge ihrer ökonomischen Wirkung ungünstig auf die Ernährung der Kinder. Dieses Moment sowohl wie die Gefahr der Ansteckung ist in verschiedenen sozialen Klassen der Bevölkerung jedenfalls von sehr verschiedenem Einfluss und man würde die Frage nach dem Bestehen noch anderweitiger Ursachen wie möglicherweise der Vererbung oder wenigstens Keimvergiftung am besten dadurch lösen, dass man eine genügende Anzahl Fälle von Tuberkulose Wohlhabender auf ihre Wirkung auf die Sterblichkeit der Kinder untersucht. Indessen ist ein solcher Versuch schwierig wegen der relativen Kleinheit des Materials.

Wenn die hohe Sterbeziffer der nachgeborenen Kinder tuberkulöser Väter nicht bloss ein Produkt des Zufalls ist, so würde sie eine Unterlage für die Annahme des Bestehens konstitutionell-hereditärer Momente bilden. Hier bedarf es jedoch noch weiterer Untersuchungen.

Es ist aber noch folgendes zu berücksichtigen: Die Zahl der Kinder (343), die im letzten Lebensjahr ihrer tuberkulösen Mutter geboren wurden, ist kaum kleiner als diejenige der (366) im letzten Lebensjahr ihres tuberkulösen Vaters Geborenen oder anders ausgedrückt, die Sterblichkeit an Tuberkulose innerhalb eines Jahres nach 
der Geburt eines Kindes ist bei beiden Geschlechtern nahezu gleich. Dies steht im Widerspruch mit der sonst grösseren 'Tuberkulosesterblichkeit auch der verheirateten Männer, lässt sich aber nach meinen Untersuchungen nicht durch eine Übersterblichkeit der verheirateten Wöchnerinnen erklären, wenn man den Einfluss des Alters und der sozialen Stellung ausschaltet. Es besteht also eine relative Untersterblichkeit, der vor kurzem Vater Gewordenen, die sich nur durch eine Auslese erklären lässt. Diese Auslese besteht darin, dass bei den Kränklichen und speziell bei schwer Kranken das Zeugungsvermögen im allgemeinen reduziert ist. Dafür spricht weiterhin die 'Tatsache, dass den $366 \mathrm{im}$ letzten Lebensjahr tuberkulöser Väter geborenen Kindern nur 103 in den letzten ${ }^{3 / 4}$ Jahren ihres Lebens Gezeugte entsprechen, während die Zahl bei gleich bleibender Zeugungskraft minhestens $3 \times 366: 4=274$ betragen müsste. Teilweise hängt dieses Verhältnis jedenfalls auch mit der geringeren Schonung zusammen, welche die tuberkulöse Frau geniesst. Demgemäss stammen die letzten Kinder tuberkulöser Väter häufiger als diejenigen tuberkulöser Mütter aus einer Zeit, in der die Tuberkulose weniger rasch vorgeschritten war, und andererseits kommen im letzten Lebensjahr des tuberkulösen Vaters und nach seinem Tod geborene Kinder häufiger auf Rechnung solcher Fälle, in welchen die Tuberkulose einen verhältnismässig akuten Verlauf nahm, während es bei akuter Tuberkulose der Mutter eher zum Abortus kommt. Nach den weiter oben mitgeteilten Zahlen entfallen auch von den Letztgeborenen tuberkulöser Mütter $73 \%$, von denen tnberkulöser Väter nur $61 \%$ auf die letzten 7 Lebensjahre. Auf Grund dieser Betrachtung lässt sich ein wesentlicher Teil des Unterschiedes in der Sterblichkeit der Kinder tuberkulöser Väter und Mütter mit einem Unterschied der angeborenen Widerstandskraft in Zusammenhang bringen, welche mit einer durchschnittlich verschieden starken Wirkung der Krankheit auf das Ei und Sperma vor der Befruchtung in denjenigen Fällen zusammenhängt, in denen das Produkt der Zeugung noch lebensfähig geboren wird. Es besteht also eine Möglichkeit den verschieden starken Einfluss der tuberkulösen Väter und Mütter auf die Sterblichkeit ihrer Kinder auch auf eine andere Ursache als den Unterschied in der Intensitität der Exposition zurückzuführen.

Jedenfalls wird aber die Nachkommenschaft, welche bei offener Tuberkulose der Eltern geboren wurde, in so erheblichem Masse schon im frühen Kindesalter dezimiert, dass man berechtigt ist zu fragen, ob sich die infektiöse Wirkung des Zusammenlebens mit tuberkulösen Eltern nicht in dieser Zeit völlig erschöpft, ob nicht alle frühzeitig Infizierten auch frühzeitig wegsterben. Fine von Todesfällen der Eltern in einem kurz zurückliegenden Zeitraum ausgehende Unter- 
suchung kann naturgemäss kein vollständiges Material für die Beantwortung dieser Frage bieten, da ein zu grosser Teil der Nachkommenschaft noch am Leben ist. Von weit zurückliegenden Todesfällen tuberkulöser Eltern kann man aber derzeit nicht leicht ausgehen, weil eine Berechnung exakter Sterbeziffern für die Gesamtbevölkerung zum Zwecke der Anstellung von Vergleichen erst seit Anfang der $70 \mathrm{er}$ Jahre des vorigen Jahrhunderts möglich ist und die Wanderungen der erwachsenen Kinder eine zu grosse und schwer kontrollierbare Rolle spielen können.

\section{Die Belastung der erwachsenen Tuberkulösen durch ihre Eltern.}

Es liegt daher näher, von den erwachsenen Tuberkulösen auszugehen und zu untersuchen, in welcher Weise sich die Sterblichkeit ihrer Eltern überhaupt und speziell an Tuberkulose verhält und wie sich ein etwaiger Einfluss elterlicher Belastung zeitlich gestaltet.

Dieser Frage bin ich in der Weise näher getreten, dass ich die elterliche Belastung der verheirateten 1883-1902 in Stuttgart an Tuberkulose einschliesslich Lungenleiden, Abzehrung und Brustleiden Gestorbenen mit der ihrer letzten Ehegatten verglich, soweit deren Eltern in den Stuttgarter Familienregistern aufgeführt waren. In diesen Fällen wurden auch die Todesursachen auswärts gestorbener Eltern durch Anfrage bei Pfarrern und Standesämtern und soweit als möglich aus den ärztlichen Leichenscheinen ermittelt; auf diese Weise wurde auch bei einem Teil der nach auswärts verzogenen Eltern noch die Todeszeit ermittelt. Dabei wurden die unehelich Geborenen durchweg von der Untersuchung ausgeschlossen.

In welchem Masse diese Methode geeignet ist, den Einfluss des sozialen und beruflichen Milieus der Abstammung auszugleichen, geht aus folgender Zusammenstellung hervor:

\section{Von 609 Tuberkulösen 595 Ehegatten}

hatten zu Vätern:

Wirto

Weingärtner, Gärtner und Bauern

Fabrikarbeiter und Taglöhner

Handwerker und Vertreter des Kleingewerbes

Niedere Angestellte

Mittlere Beamte und nicht akademisch gebildete Lehrer

Kaufleute

$$
\begin{array}{rlrl}
27 & =4,4 \% & 30 & =5,1 \% \\
92 & =15,1 \% & 90 & =15,1 \% \\
109 & =17,9 \% & 95 & =16,0 \% \\
268 & =44,0 \% & 275 & =46,2 \% \\
18 & =3,0 \% & 20 & =3,4 \% \\
19 & =3,1 \% & 22 & =3,7 \% \\
30 & =5,9 \% & 21 & =3,5 \% \\
40 & =6,6 \% & 37 & =6,2 \% \\
6 & =1,0 \% & 5 & =0,8 \% .
\end{array}
$$

Der Beruf blieb fraglich bei 
Die Unterschiede in der prozentualen Verteilung der einzelnen verschiedenen Kategorien sind fast durchweg gering. Wenn die Fabrikarbeiter und Taglöhner etwas stärker unter den Eltern vertreten sind, so ist es umgekehrt bei den Vertretern des Handwerks; unter diesen sind zahlreiche Gehilfen, welche sozial mit dem Fabrikarbeiter auf einer Stufe stehen, ihre Zahl war aber nicht genau zu ermitteln. Beide Kategorien zusammengefasst ergaben beiderseits nahezu dieselben Prozentsätze (61,9 und 62,2). Die Berufe, welche besonders leicht zu Tuberkulose führen und teilweise infolge der Ausübung in der Wohnung besonders gefährlich sind, sind bei den Eltern der Tuberkulösen nich twesentlich stärker, teilweise sogar schwächer vertreten als bei ihren Schwiegereltern, so fand ich bei den

der Tuberkulösen

$\begin{array}{lr}\text { Schneider } & 22 \\ \text { Schuhmacher } & 19 \\ \text { Schreiner } & 26 \\ \text { Steinhauer und Maurer } & 13 \\ \text { Sattler } & 7 \\ \text { Buchdrucker } & 8\end{array}$

Vätern Schwiegervätern

$\begin{array}{rr}22 & 19 \\ 19 & 18 \\ 26 & 22 \\ 13 & 25 \\ 7 & 6 \\ 8 & 9\end{array}$

Von seiten der sozialen Stellung und des' Berufes konnte also bei den Eltern der Tuberkulösen eine stärkere Vertretung der Tuberkulose nicht erwartet werden.

Weiterhin lässt sich nachweisen, dass auch die Geburtszeit der Tuberkulösen, ihrer Eltern und Schwiegereltern und die sich hieraus ergebende Ziffer für Alter der Eltern und Schwiegereltern bei der Geburt der in Frage kommenden Kinder und die Dauer ihrer Beobachtung bis Ende 1902 keine erheblichen Unterschiede aufwiesen.

Es waren nämlich geboren von

$$
\begin{array}{llll}
609 & 611 & 595 & 600
\end{array}
$$

Vätern Müttern Schwiegervätern Schwiegermüttern der Tuberkulösen

$\begin{array}{rrrrr}\text { 1743-1752: } & - & - & 1 & - \\ \text { 1753-1762: } & - & - & - & 2 \\ \text { 1763-1772: } & 3 & 2 & 2 & 3 \\ 1773-1782: & 13 & 6 & 12 & 5 \\ 1783-1792: & 36 & 25 & 41 & 23 \\ 1793-1802: & 70 & 56 & 61 & 51 \\ 1803-1812: & 140 & 129 & 126 & 113 \\ 1813-1822: & 157 & 170 & 143 & 147 \\ 1823-1832: & 105 & 107 & 109 & 125 \\ 1833-1842: & 62 & 80 & 77 & 94 \\ 1843-1852: & 22 & 35 & 22 & 35 \\ 1855-1862: & 1 & 1 & 1 & 2\end{array}$


Einige Fälle ohne ermittelte Geburtsjahre sind nicht gezählt, daher die Unterschiede in den Zahlen für beide Geschlechter. bei den

Der Geburtstag war von dem Ende des Jahres 1902 entfernt $\begin{array}{ccc}\text { Vätern Müttern } & \begin{array}{c}\text { Schwieger- } \\ \text { vätern }\end{array} & \begin{array}{c}\text { Schwieger- } \\ \text { müttern }\end{array}\end{array}$

der Tuberkulösen insgesamt 52693 Jahre 50862 Jahre 50717 Jahre 49264 Jahre durchschnittlich 86,5 " $83,2, \quad 85,3$ " $\quad 82,1$,

Das Alter betrug bei der Geburt der

Tuberkulösen

Ehegatten Tuberkulöser für deren

Väter Mütter

Schwiegerväter

Schwiegermütter insgesamt 21186 Jahre 19080 Jahre 20552 Jahre 18861 Jahre durchschnittlich $34,8 \quad, \quad 31,2 \quad$ " $34,5 \quad$, $31,4 \quad$,

Die Eltern der Tuberkulösen sind also mit demselben Alter statistisch in Beobachtung getreten wie deren Schwiegereltern.

Aus dem Unterschied der durchschnittlichen Geburtszeit der Eltern und Kinder ergaben sich als Beobachtungsdauer bis Ende 1902 für die

Väter Mütter $\begin{gathered}\text { Schwieger- } \\ \text { väter }\end{gathered} \quad \begin{gathered}\text { Schwieger- } \\ \text { mütter }\end{gathered}$

insgesamt 31507 Jahre 31782 Jahre 30165 Jahre 30403 Jahre durchschnittlich $51,7 \quad, \quad 52,0 \quad, \quad 50,8 \quad, \quad 50,7 \quad$ "

Da die Eltern etwas länger in Beobachtung waren als die Schwiegereltern, so muss bei ihnen auch eine etwas grössere relative Zahl von Todesfällen vermutet werden. Da indes die Beobachtung sich fast bei allen Individuen bis zum 60. Lebensjahre erstreckt, so kommt dieser Fehler erst jenseits dieses Alters wesesentlich in Betracht, also in einem Alter, in dem die Tuberkulose keine allzugrosse Rolle mehr spielt.

Die Zahl der Todesfälle betrug, mit Ausnahme von drei bezw. vier nicht bezüglich des Alters festgestellten,

$\begin{array}{crc}\text { im Alter bei den } 1220 \text { Eltern } & 1195 & \text { Schwiegereltern } \\ \text { bis } 40 \text { Jahren } & 78 & 66 \\ 40-60 \quad " & 374 & 354 \\ 60 \text { und mehr " } & 549 & 521\end{array}$

oder auf je 100 überhaupt beobachtete Personen 


$\begin{array}{rrc}\text { im Alter } & \text { Eltern } & \text { Schwiegereltern } \\ \text { bis zu } 40 \text { Jahren } & 6,4 \% & 5,5 \% \\ \text { von } 40-60 \quad, & 30,7 \% & 29,7 \% \\ \text { " über } 60 \quad, & 45,1 \% & 43,6 \% \\ \text { insgesamt } & 82,2 \% & 78,7 \%\end{array}$

Die Sterblichkeit der Eltern verhält sich zu denjenigen der Schwiegereltern

$$
\begin{aligned}
& \text { im Alter bis } 40 \text { Jahren wie 116:100 }
\end{aligned}
$$

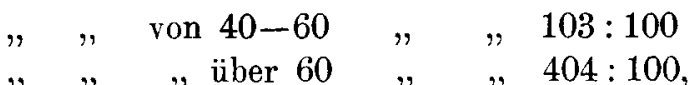

der Unterschied würde auch in den höheren Altersklassen grösser erscheinen, wenn die Todesfälle mit den zu Anfang jeden Alters Lebenden verglichen wären. Indessen beruht der gefundene Unterschied auf einer Differenz von 32 Fällen im Alter bis zu 60 Jahren, während der mittlere Fehler etwa 21 beträgt, demnach kann der Zufall den Unterschied in der Sterblichkeit der Eltern und Schwiegereltern ebensowohl zu gross wie zu klein erscheinen lassen.

Ein prägnanteres Ergebnis erhält man jedoch bei Feststellung der Todesfälle an Tuberkulose bei Eltern und Schwiegereltern der Tuberkulösen.

Die absoluten Zahlen sind

bei den Vätern der Tuberkulösen 75 Fälle sicherer, 43 Fälle wahrscheinlicher Tuberkulose,

bei den Müttern der Tuberkulösen 24 Fälle sicherer, 41 Fälle wahrscheinlicher Tuberkulose,

bei den Schwiegervätern der Tuberkulösen 51 Fälle sicherer, 28 Fälle wahrscheinlicher Tuberkulose,

bei den Schwiegermüttern der Tuberkulösen 17 Fälle sicherer, 25 Fälle wahrscheinlicher Tuberkulose,

es kommen also auf 1220 Eltern

$8,1 \%$ sichere, $6,9 \%$ wahrscheinliche Tuberkulose, auf 1195 Schwiegereltern

$5,7 \%$ sichere, $4,4 \%$ wahrscheinliche Tuberkulose, insgesamt stehen $15,0 \%$ bei den Eltern gegenüber $10,1 \%$ bei den Schwiegereltern.

Bei gesonderter Untersuchung beider Geschlechter erhält man

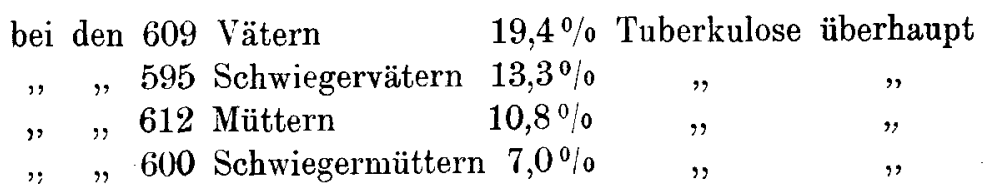


Es verhält sich also die Tuberkulose-Sterblichkeit

der Eltern Tuberkulöser zu derjenigen ihrer Schwiegereltern wie $15,0: 10,1$ oder wie $150: 100$,

der Väter Tuberkulöser zu derjenigen ihrer Schwiegereltern wie $19,4: 13,3$ oder wie $146: 100$,

der Mütter Tuberkulöser zu derjenigen ihrer Schwiegermütter wie $10,6: 7,0$ oder wie $152: 100$.

Die Häufigkeit sicherer Fälle von Tuberkulose verhält sich für beide Geschlechter zusammen wie

diejenige unsicherer Fälle wie

$$
8,1: 5,7 \text { oder wie } 142: 100 \text {, }
$$

$$
6,9: 4.4 \text { oder wie } 157: 100 \text {. }
$$

Die Tuberkulose-Sterblichkeit der Eltern Tuberkulöser erscheint um $49 \%$, bei Berücksichtigung nur sicherer Fälle um $40 \%$ höher als diejenige ihrer Schwiegereltern. Dass auch hier der Einfluss der Mutter etwas grösser ist als derjenige des Vaters, mag Zufall sein. Der absolute Zahlenunterschied beträgt 62, während der mittlere Fehler etwa 11 beträgt. Dieses Resultat ist also weit sicherer als dasjenige des Einflusses der familiären Beziehungen zu Tuberkulose auf die Gesamtsterblichkeit.

Vernachlässigt man die Fälle von Tuberkulose beider Eltern, so erhält man die Häufigkeit der Belastung durch eines oder das andere der Eltern, indem man obige Zahlen mit 2 multipliziert. Es waren also 30,0\% Tuberkulöser elterlicherseits belastet gegen $20,2 \%$ ihrer Ehegatten. Unter den Ehegatten der Tuberkulösen ist auch eine Anzahl bereits an Tuberkulose gestorbener Personen und eine weitere Anzahl hat dies Schicksal noch zu erwarten. Die gefundenen Ergebnisse deuten also darauf hin, dass der Unterschied der Belastung Tuberkulöser und Nichttuberkulöser noch stärker ist als derjenige der Zahlen 3 und 2.

Nun ist weiterhin zu berücksichtigen, dass die Verheirateten bereits eine gesundheitlich günstige Auslese darstellen; ein Teil derjenigen Belasteten, welche bereits tuberkulös sind oder deren Konstitution die Ehe verbietet, kommt überhaupt nicht zur Eheschliessung. Eine Untersuchung, welche sich auf alle Erwachsenen erstrecken würde, müsste daher die Tuberkulosesterblichkeit der Belasteten gegenüber derjenigen der Nichtbelasteten noch erheblich höher erscheinen lassen als dies das Verhältnis 3:2 zum Ausdruck bringt.

Für die oben unterschiedenen Berufsklassen haben sich ziemliche Unterschiede in dem Einfluss der Belastung ergeben. Fasst man Arbeiter, Handwerker, niedere Angestellte und Wirte als eine Gruppe 
zusammen, in welcher die ungesunden und bezüglich der Ansteckung mit Tuberkulose besonders gefährlichen Berufe enthalten sind, und vernachlässigt man den Unterschied in der Zahl der Väter und Mütter, so erhält man

auf $2 \times 429$ Eltern aus diesen Berufskreisen 131 oder $15,3 \%$, auf $2 \times 425$ Schwiegereltern hingegen 96 oder 11,3\% Tuberkulose, oder ein Verhältnis der Belastung wie 135̃: 100, hingegen für die übrigen Berufsgruppen

auf $2 \times 181$ Eltern 54 Fälle von Tuberkulose oder 14,9\%, auf $2 \times 170$ Schwiegereltern nur 25 oder $7,3 \%$; somit ein Verhältnis von 204:100.

In der sozial und beruflich günstiger gestellten zweiten Gruppe erscheint also der Einfluss der Belastung stärker, obgleich hier der Einfluss der sozialen Verhältnisse, des intimeren Zusammenlebens, geringer ist. Das scheint im Sinne der Vererbung zu sprechen, bedarf aber weiterer Bestätigung durch grösseres Material aus den Kreisen der Wohlhabenden.

Ich habe nun untersucht, in welcher Beziehung die Tuberkulosetodesfälle der Eltern und Schwiegereltern zu denen der Tuberkulösen beziehungsweise zu ihrem und ihrer Ehegatten Alter stehen.

Der Zeitraum zwischen dem Tod der Tuberkulösen und dem ihrer tuberkulösen Eltern betrug bei 183 Fällen ingesamt 4811, durchschnittlich also 26,3 Jabre, während der Tod der tuberkulösen Schwiegereltern von dem erstgenannten Zeitpunkt in 121 Fällen, zusammen 3245, durchschnittlich 26,8 Jahre entfernt war. 7 der Eltern starben zusammen 43 Kalenderjahre, 4 der Schwiegereltern zusammen 13 Kalenderjahre nach den Tuberkulösen; rechnet man diese Fälle $\mathrm{ab}$, so starben in den übrigen 176 bezw. 117 Fällen die tuberkulösen Eltern der Tuberkulösen 27,1 Jahre, die tuberkulösen Schwiegereltern 27,6 Jahre vor dem Tode der Tuberkulösen selbst. Will man alle Fälle des Zusammentreffens von Tuberkulose eines verheirateten Erwachsenen mit Tuberkulose seiner Eltern auf Infektion zurückzuführen, so ergibt sich ein ziemlich langer Zeitraum der Latenz und weiterhin kein wesentlicher Unterschied gegenüber der zeitlichen Entfernung des Todes der tuberkulösen Schwiegereltern. Daraus musste man auf eine ziemlich gleichmässige Verteilung der Häufigkeit der Todesfälle von Eltern und Schwiegereltern schliessen. Das Ergebnis gestaltet sich aber einigermassen anders; wenn man die verschiedene Häufigkeit dieser Zeiträume zwischen Tod der Tuberkulösen und ihren Eltern bezw. Schwiegereltern tatsächlich untersucht. 
Es kamen nämlich auf

1220 Eltern

1195 Schwiegereltern

Todesfälle an Tuberkulose im gleichen Kalenderjahr mit dem der Tuberkulösen

1-4 Kalenderjahre früher als letztere $5-9$

$10-19$

$20-29$ 30 u. mehr $1-19$

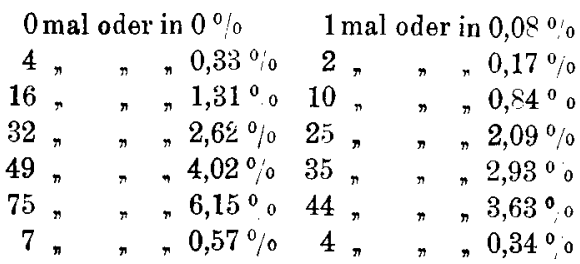

Die Häufigkeit der Fälle von Tuberkulose der Eltern verhält sich also zu denjenigen der Schwiegereltern, sofern die Eltern vor den Kindern starben, bei einer Zeitdifferenz von

$\begin{array}{ccc}0-9 \text { Jahren } & \text { wie } 150: 100 \\ 10-19, " & " & 125: 100 \\ 20-29, & " & 137: 100 \\ 30 \text { und mehr Jahren } & " & 167: 100 . \\ \text { bei späterem Tod der Eltern } & , & 168: 100 .\end{array}$

Bei jedem Zeitraum weisen die Eltern der Tuberkulösen eine Übersterblichkeit an Tuherkulose auf.

Bei den kurzen und langen Zwischenräumen erhält man aber eine grössere Übersterblichkeit der Eltern an Tuberkulose im Vergleich mit den Schwiegereltern als bei den mittleren und die grösste Differenz fällt auf die auch numerisch am stärksten vertretenen langen Zwischenräume. Die Fälle mit kurzen $Z$ wischenräumen fallen numerisch wenig ins Gewicht, hier mag der Zufall demnach eine zu starke Differenz vortäuschen. Jedenfalls kann die Mehrbelastung der Tuberkulösen durch ihre Eltern nur zu sehr geringem Teil durch eine häufigere kurze \%eit zurückliegende Infektionsgelegenheit seitens der Eltern erklärt werden. Die Hauptmasse des gefundenen Unterschiedes fällt zu Lasten einer lange zurückliegenden Tuberkulose der Eltern.

Noch in anderer Weise werden diese Beziehungen illustriert durch eine Untersuchung, wie lange nach dem Tode tuberkulöser Eltern und Schwiegereltern die belasteten Tuberkulösen und deren meist nicht tuberkulöse Ehegatten geheiratet haben. Der Tod der tuberkulösen Eltern ging der Eheschliessung der 183 belasteten Tuberkulösen um 1770 Kalenderjahre, also durchschnittlich 9,7 Jahre voraus, der Tod der tuberkulösen Schwiegereltern bei den belasteten Ehegatten um 1277, durchschnittlich also um 10,5 Jahre voraus. Rechnet man die 34 bezw. 20 Fälle ab, in welchen die Tuberkulösen ein oder mehrere Kalenderjahre vor dem Tod ihrer tuberkulösen Eltern und Schwiegereltern starben, so erhält man für die Fälle, in denen sie vor der Eheschliessung ihrer Kinder starben, 2008 und 1439 oder durchschnittlich 14,3 und 14,8 Jahre. 
In diesen letzteren Fällen starben die

Eltern Schwiegereltern der Tuberkulösen

im Kalenderjahre der Eheschliessung $9 \mathrm{mal}$ $4 \mathrm{mal}$

" 1 -4 Kalenderjahr v. d. Eheschliessg.

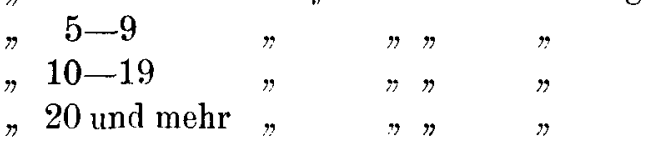

$\begin{array}{ll}17 \% & 14 " \\ 16 " & 20 " \\ 68 " & 36 " \\ 39 " & 27 "\end{array}$

Auf die 1220 Eltern und 1195 Schwiegereltern berechnet, verhält sich deren Sterblichkeit

1 und mehr Kalenderjahre nach der Eheschliessung ihrer Kinder wie $2,79 \%: 1,67 \%$ oder wie $167: 100$;

0-9 Kalenderjahre vor der Eheschliessung ihrer Kinder wie 3,44\%: $\%: 18 \%$ oder wie $108: 100$,

10 und mehr Kalenderjahre vor der Eheschliessung ihrer Kinder wie $8,77 \%: 5,27 \%$ oder wie $166: 100$.

Auch hier weisen die extremen Fälle den grössten Unterschied in der 'Tuberkulosesterblichkeit der Eltern und Schwiegereltern auf; das Resultat bezüglich der lange vor der Eheschliessung erfolgten Todesfälle der Eltern und Schwiegerelten steht dabei auf einer breiteren numerischen Basis als bezüglich der nach der Eheschliessung gestorbenen. Inwieweit dieses letztere auf ein häufiges Zusammenleben mit den Eltern nach der Ehe zurückzuführen ist, muss vorlïufig fraglich bleiben. Jedenfalls erklärt diese Möglichkeit nur einen kleinen Teil des Überschusses der Tuberkulosesterblichkeit der Eltern Tuberkulöser, während deren Hauptanteil auf die Eltern fällt, die lange Zeit vor der Eheschliessung bereits an Tuberkulose sterben.

Der von Cornet gestellten Forderung, es sollen nur solche Fälle untersucht werden, die nicht in Berührung mit kranken Familienangehörigen waren, genügt nach diesem Ergebnis mein Material zum grösseren Teil, wenn man die Cornetsche Ansicht von der meist kurzen Dauer der Infektion akzeptiert. Ob dies angeht, ist aber eben der strittige Punkt des gegenwärtigen Kampfes um die Ätiologie der Tuberkulose. Geht es aber nicht an, so ist auch die Forderung Cornets praktisch so gut wie unerfüllbar.

Fragt man sich endlich, wie häufig in verschiedenem Alter ein Zusammenleben mit hochgradig tuberkulösen Eltern stattfand, beziehungsweise in welchem Alter der Kinder der 'Tod ihrer tuberkulösen Eltern stattfand, so erhält man folgendes Ergebnis: 
Es starben an Tuberkulose von den 1220 Eltern 1195 Schwiegereltern

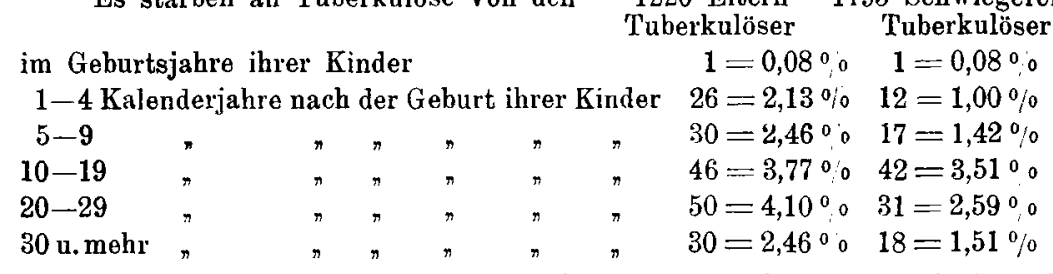

und das Verhältniss der Tuberkulosesterblichkeit der Eltern und Schwiegereltern beträgt

\begin{tabular}{|c|c|c|c|}
\hline \multirow{2}{*}{\multicolumn{3}{|c|}{$\begin{array}{l}\text { im Geburtsjahre ihrer Kinder } \\
1-4 \text { Kalenderjahre später }\end{array}$}} & $100: 100$ \\
\hline & & & $213: 100$ \\
\hline $5-9$ & $\pi$ & $n$ & $173: 100$ \\
\hline $10-19$ & $\pi$ & $\pi$ & $107: 100$ \\
\hline $20-29$ & 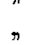 & 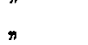 & $159: 100$ \\
\hline 30 u. mohr & $\pi$ & $\pi$ & $163: 100$. \\
\hline
\end{tabular}

Auch hier zeigen, wenn man von der Sterblichkeit im Geburtsjahr der Kinder absieht, die Extreme der Zeitintervalle zwischen Geburt der Kinder und Tod der Eltern wieder die stärksten Unterschiede in der Tuberkulosesterblichkeit der Eltern und Schwiegereltern, dabei sind die Unterschiede bei frühem Tod der Eltern zwar stärker, stehen aber numerisch auf weniger breiter Basis als die bei spätem Tod der Eltern. Ob die geringen Unterschiede der Sterblichkeit der Eltern und Schwiegereltern im 10.-19. Kalenderjahre nach der Geburt ihrer Kinder auf Zufall beruhen, muss vorläufig dahingestellt bleiben. Auffallend ist die überaus schwache Vertretung der Kalenderjahre der Geburt der Kinder. Diese Tatsache stimmt mit der oben ausgesprochenen Vermutung überein, dass Kinder, welche im letzten Lebensjahr ihrer tuberkulösen Eltern geboren worden, und somit frühzeitig sehr stark der Infektion ausgesetzt waren, selten in das Alter der Erwachsenen gelangen, weil sie im Kindesalter zu stark dezimiert wurden.

Wir kommen daher zu folgendem Ergebnis. Will man die Übersterblichkeit der Eltern tuberkulöser Erwachsener an Tuberkulose lediglich auf vermehrte Infektionsgelegenheit in der Familie zurückführen, so muss man in einem grossen Teil der Fälle eine sehr lange Dauer der Infektion annehmen, will man weiterhin alle diese Fälle durch Infektion im Säuglingsalter erklären, so muss man bei deren Eltern häufig eine sehr lange Dauer der offenen Tuberkulose annehmen.

Die ganze Übersterblichkeit der Eltern auf Infektion im Kindesalter zurückzuführen ist sehr schwierig, da auch noch mehr als 30 Jahr nach der Geburt ihrer Kinder die Eltern Tuberkulöser eine erhöhte Tuberkulosesterblichkeit aufweisen. Mit einer durchschnitt- 
lich kurze Zeit zurückliegenden Infektionsmöglichkeit in jedem Alter lässt sich die Übersterblichkeit der Belasteten an Tuberkulose ebenfalls nicht vollständig erklären. Man muss daher mit einer ziemlichen Häufigkeit sehr langer Dauer der Latenz der Tuberkulose rechnen. So lange diese nicht als häufig vorkommend erwiesen ist, können auch andere Faktoren für die Übersterblichkeit der Eltern Tuberkulöser an Tuberkulose in Betracht gezogen werden und bis dahin ist die Möglichkeit erblicher Neigungen nicht ausgeschlossen.

A priori ist eine langdauernde Wirkung erblicher Vorgänge jedenfalls leichter verständlich als die einer lange latent gebliebenen Infektion. Immerhin führt die Lehre von der langen Latenz der Tuberkulose zu sonderbaren Ergebnissen. Vergleicht man den Einfluss der Belastung auf die 'Tuberkulosesterblichkeit der Verheirateten mit demjenigen des ehelichen Zusammenlebens mit Tuberkulösen, so findet man bei Personen, die durchschnittlich etwa 10 Jahre nach dem Tod ihrer tuberkulösen Eltern heirateten, eine Tuberkulosesterblichkeit von über $150 \%$ der Erwartung, hingegen bei den überlebenden Ehegatten Tuberkulöser fünf und mehr Jahre nach dem Todesfall $180 \%$ ohne Berücksichtigung des sozialen Faktors, mit Berücksichtigung desselben ebenfalls nur $150 \%$ der Erwartung. Dabei zeigt auch noch eine zeitlich sehr lange zurïckliegende Belastung einen grossen numerischen Einfluss. Will man diesen lediglich auf latente Infektion zurückführen, so gelangt man zu dem paradoxen Ergebnis, dass eine lange Zeit latent gebliebene Infektion mindestens denselben numerischen Einfluss hat wie die Infektionsgelegenheit durch einen kranken Ehegatten. Im Sinne der Lehre Behrings und seiner Nachfolger könnte dies Paradoxon wohl nur dadurch unschädlich gemacht werden, dass man dem Zusammenleben mit tuberkulösen Ehegatten lediglich einen stark disponierenden Einfluss zuschreibt.

\section{Die Belastung durch tuberkulöse Geschwister.}

Zur Vollständigkeit der Untersuchung gehört auch die Kenntnis der Beziehungen der Tuberkulösen zu tuberkulösen Geschwistern. Diese habe ich in ähnlicher Weise wie die der Eltern Tuberkulöser durch Vergleich der Sterblichkeit der ehelichen Geschwister von 280 ehelich geborenen 1893-1902 gestorbener Tuberkulösen mit derjenigen der entsprechenden Geschwister von 258 Ehegatten Tuberkulöser untersucht. Die 280 Tuberkulösen hatten zusammen 1475 oder durchschnittlich 5,3, die 258 Ehegatten Tuberkulöser zusammen 1445 oder durchschnittlich 5,6 Geschwister. Die grosse Zahl der Geschwister auf beiden Seiten stellt im wesentlichen den mathematischen 
Ausdruck der Tatsache dar, dass verhältnismässig mehr Menschen aus grossen als aus kleinen Familien stammen, wie weit daneben soziale Verhältnisse ein Rolle spielen, lässst sich nicht nachweisen.

Die etwas geringere Geschwisterzahl der Tuberkulösen kann auf Zufall beruhen, aber auch damit zusammenhängen, dass ihre Eltern häufiger im zeugungsfähigem Alter gestorben sind als diejenigen der Ehegatten Tuberkulöser ${ }^{1}$ ).

Es waren

lebendgeboren totgeboren

von den

Brüdern

der Tuberkulösen

731

57

Schwestern

Schwägern " "

649

38

Schwägerinnen

$"$

744

37

633

31

Die Totgeburtenziffer betrug bei den
Geschwistern der Tuberkulösen
$6,4 \%$
bei denen ihrer Ehegatten
$4,7 \%$

war also bei ersteren nicht unerheblich höher; teilweise mag dies mit hüufigeren Frühgeburten in ersteren Familien zusammenhängen.

Von den Lebendgeborenen sind geboren

Brüder Schwestern Schwäger Schwägerinnen

der Tuberkulösen

$\begin{array}{rrrrr}1803-1812: & - & - & 3 & 5 \\ 1813-1822: & 4 & 6 & 5 & 5 \\ 1823-1832: & 50 & 43 & 40 & 29 \\ 1833-1842: & 94 & 77 & 84 & 80 \\ 1843-1852: & 160 & 134 & 158 & 120 \\ 1853-1862: & 141 & 129 & 133 & 140 \\ 1863-1872: & 173 & 151 & 171 & 133 \\ 1873-1882: & 89 & 107 & 125 & 102 \\ 1883-1892: & \left.20^{2}\right) & \left.12^{2}\right) & \left.24^{2}\right) & \left.18^{2}\right) \\ 1893-1902: & - & - & \left.1^{2}\right) & \left.1^{2}\right)\end{array}$

Die durchschnittliche Entfernung des Geburtstags vom Ende der Beobachtung, dem 31. Dezember 1902, betrug bei den

\begin{tabular}{|c|c|c|}
\hline Brüdern & der 'Tuberkulösen & 46,4 Jahre \\
\hline Schwestern & $\eta$ & 46,3 \\
\hline Schwägern & ״ & 44,8 \\
\hline Schwägerinnen & $"$ & 45,1 \\
\hline
\end{tabular}

1) Gottstein hat bei den aus Ehen mit nur 1-2 Kindern stammenden Versicherten eine auffallend grosse Tuberkulosesterblichkeit gefunden.

2) Von den nach 1887 geboreuen Geschwistern der Tuberkulösen bezw. ihrer Ehegatten lebten Ende 1902 noch 2 bezw. 5 . 
Die etwas längere Beobachtuigsdauer der Geschwister der Tuberkulösen war geeignet, ihre Tuberkulosesterblichkeit etwas zu hoch erscheinen zu lassen.

Von den Lebendgeborenen starben im ersten Lebensjahr

197 Brüder

149 Schwestern

223 Schwägern
$=27 \%$

$=23 \%$

$=30 \%$

346 Geschwist. Tuberkulös. $=25 \%$

379 Geschwister der Ehegatten Tuberkulöser $=27 \%$

Demnach erscheint die Sterblichkeit des ersten Lebensjahres bei den Geschwistern der Tuberkulösen sogar etwas günstiger als bei denen ihrer Ehegatten. Aus den Darlegungen des ersten Abschnittes geht hervor, dass dadurch der ungünstige Einfluss der infektiösen Verwandtschaft auf die Kindersterblichkeit nicht widerlegt wird, es ist aber begreiflich, dass er bei den Geschwistern Erwachsener weniger stark zum Ausdruck kommt, da letztere eine Auslese aus den weniger ungünstigen Familien darstellen, damit ist Zufälligkeiten ein grösserer Spielraum eingeräumt.

Über das Schicksal der über ein Jahr alt gewordenen Personen konnte folgendes ermittelt werden.

Es starben a) ledig und verheiratet in Stuttgart:

b) ledig auswärts :

\begin{tabular}{|c|c|c|c|c|c|c|c|c|}
\hline \multirow[t]{2}{*}{ Alter } & \multicolumn{2}{|c|}{ Brüder } & \multicolumn{2}{|c|}{$\begin{array}{l}\text { Schwestern } \\
\text { zulösen }\end{array}$} & \multicolumn{2}{|c|}{$\begin{array}{c}\text { Brüder } \\
\text { der Whegatte }\end{array}$} & \multicolumn{2}{|c|}{$\begin{array}{l}\text { Schwestern } \\
\text { Tuberkulöser }\end{array}$} \\
\hline & a & $\mathrm{b}$ & $\mathbf{a}$ & $\mathrm{b}$ & $\mathbf{a}$ & $\mathrm{b}$ & $a$ & $\mathrm{~b}$ \\
\hline 1-4 Jahre & 59 & 5. & 47 & 5 & 41 & 1 & 58 & 2 \\
\hline $5-9$ & 13 & - & 7 & 2 & 19 & $\cdots$ & 5 & - \\
\hline $10-14$ & 3 & 2 & 5 & 1 & 6 & 1 & 3 & 一 \\
\hline $15-19$ ? & 12 & - & 14 & 1 & 10 & 2 & 9 & - \\
\hline $20-29$ & 27 & 11 & 26 & 3 & 19 & 1 & 20 & 3 \\
\hline $30-39$ & 35 & 1 & 17 & 2 & 26 & 1 & 15 & 1 \\
\hline $40-49$ & 7 & 2 & 15 & 1 & 19 & - & 6 & 2 \\
\hline $50-59$ & 15 & 1 & 9 & 1 & 8 & 1 & 8 & 2 \\
\hline $60-69$ & 13 & 1 & 4 & - & 9 & 1 & 10 & - \\
\hline $70-79$ & .4 & - & 3 & - & 3 & - & 5 & - \\
\hline $\begin{array}{l}80-99 \\
\text { in unbekann- }\end{array}$ & - & - & - & - & 3 & - & 2 & - \\
\hline tem Alter & - & 1 & - & 1 & - & - & - & - \\
\hline insge & 188 & 24 & 147 & 17 & 163 & 8 & 141 & 10 \\
\hline
\end{tabular}


Es schieden mit ihrer Verheiratung nach auswärts aus der Beobachtung

60 Brïder,

32 Schwestern,
32 Schwäger,

31 Schwägerinnen.

Die grössere Anzahl nach auswärts verzogener Geschwister Tuberkulöser ist geeignet, deren allgemeine und Tuberkulosesterblichkeit zu gering erscheinen zu lassen im Vergleich mit den Geschwistern ihrer Ehegatten.

Es treffen, soweit bekannt, auf die das erste Lebensjahr überlebenden

1034 Geschwister Tuberkulöser $\quad 376=36,4 \%$ Todesfälle $998 \quad " \quad$ der Ehegatten Tuberkulöser $322=32,3 \%$ "

Auf das 2.-15. Lebensjahr treffen

149 Todesfälle an Geschwistern Tuberkulöser $=14,46 \%$

$136 \quad " \quad " \quad$ ihrer Ehegatten $=13,63 \%$

der über ein Jahr alt Gewordenen. 2 bezw. 5 hatten am Ende des Jahres 1902 das 15 . Lebensjahr noch nicht vollendet.

Von den über 15 Jahre alt gewordenen 883 bezw. 858 Geschwistern der

Tuberkulösen Ehegatten Tuberkulöser starben im $16-50$ Lebensjahr $171=19,71 \% \quad 134=15,64 \%$

$$
\begin{aligned}
& " \quad, 51-100 \quad \text { " } \quad 51=5,78 \% \quad 52=6,07 \% \\
& " \quad, 16-100 \quad " \quad 225=25,48 \% \quad 186=21,70 \%
\end{aligned}
$$

Die allgemeine Sterbeziffer der Geschwister der Tuberkulösen war bis zum 50. (bezw. 60.) Lebensjahr derjenigen der Geschwister ihrer Ehegatten gegenüber erhöht. Nach dieser Zeit ist sie scheinbar nicht mehr erhöht, in Wirklichkeit würde sich aber eine Erhöhung auch für dieses Alter ergeben, wenn man die Zahl der Todesfälle nach dem 50. bezw. 60. Lebensjahre mit der Zahl der zu Anfang des 51. bezw. 61. Lebensjahre Lebenden vergleichen würde. Das war aber mit Rücksicht auf das unbekannte Schicksal der nach auswärts verzogenen nicht genau möglich.

Von den Todesfällen im 2.-15. Lebensjahr betrafen Tuberkulose

bei den Geschwistern der Tuberkulösen $26=2,6 \%$

" " " "Ehegatten Tuberkulöser $19=1,9 \%$ der über ein Jahr alt Gewordenen. Darunter sind aber nur 12 bezw. 11 sichere Fälle. Die grosse Zahl der unsicheren Fälle hängt mit der ungenauen Todesursachenstatistik des Kinderalters namentlich in frühen Zeiten zusammen.

Die Tuberkulosesterblichkeit der Geschwister Tuberkulöser in diesem Alter verhält sich zu denjenigen der Geschwister ihrer Ehegatten wie $137: 100$. 
Auf die über 15 Jahre alt Gewordenen treffen Fälle von Tuberkulose

bei den Geschwistern der Tuberkulösen $89=10,1 \%$

" $"$ ", Ehegatten Tuberkulöser $50=5,8 \%$ darunter 15 bezw. 7 unsichere Fälle.

Die Tuberkulosesterblichkeit der Geschwister Tuberkulöser in diesem Alter verhielt sich zu derjenigen der Geschwister ihrer Ehegatten ohne unsichere Fälle wie 180:100

mit unsicheren Fällen „, $168: 100$.

Diese sehr zuverlässige Statistik ergibt also bei den über 15 Jahre alten Geschwistern eine stärkere Wirkung der Belastung durch tuberkulöse Geschwister wie die Wirkung elterlicher Belastung in Abschnitt II. Die auf weniger sicherem Material beruhenden Ergebnisse bei den Eltern der Tuberkulösen werden also durch die befriedigend exakte Statistik der Geschwister vollauf bestätigt.

Auch hier fand sich meist ein ziemlich grosser Zeitraum zwischen dem Tod zweier zusammengehörigen Geschwister an Tuberkulose, 79 mal betrug ęr mehr als 7 Jahre, von den 36 Fällen mit kurzem Zeitraum war in 21 mindestens eines der Geschwister beim Tode des anderen bereits verheiratet, nur in 15 Fällen $=13 \%$ war ein Zusammenleben mit tuberkulösen Geschwistern kurze Zeit vor dem eigenen Tod der verheirateten Tuberkulosen einigermassen wahrscheinlich. Die tuberkulösen Geschwister der Ehegatten Tuberkulöser starben in 50 Fällen mehr als 7 Kalenderjahre vor letzteren, unter den übrigen 19 sind nư 5, bei denen ein Zusammenleben mit den Ehegatten der Tuberkulösen in den letzten 7 Jahren des Lebens der letzteren wahrscheinlich ist. Ein Zusammenleben mit tuberkulösen Geschwistern kurze Zeit vor dem Tode hatte also bei den verheirateten Tuberkulösen etwa 3 mal so häufig stattgefunden, als bei ihren Ehegatten. Aber dieser Unterschied reicht lange nicht aus zur Erklärung der ganzen Übersterblichkeit der Geschwister Tuberkulöser, die auch bei denen erhöht war, die kurze Zeit vor dem Tode nicht mit ihren Geschwistern zusammenlebten. In der überwiegenden Mehrzahl der Fälle muss man auch hier mit einer langen Latenz der Infektion rechnen, wenn man die ganze Übersterblichkeit der Geschwister Tuberkulöser nur auf Infektion in der Familie zurückführen will.

Dieses Resultat steht in schroffem Gegensatz zu den Resultaten Boegs der in fast allen Fällen von Belastung mehrerer Mitglieder einer Familie auch Infektionsgelegenheit in der Familie konstatierte. Der Unterschied rührt daher, dass B o e g alle Fälle von Tuberkulose berücksichtigt hat ohne Rücksicht auf Alter und Familienstand und 
dass er die Dauer von der Infektionsgelegenheit bis zum Tode nicht berücksichtigte.

Vom Standpunkt des Statistikers lässt sich auch bei einem grossen Zeitraum zwischen den Todesdaten zweier zusammengehöriger Familienmitglieder die Infektionsmöglichkeit nicht ausschliessen. Aus diesem Grund ist es vorerst nicht möglich, nit Hilfe der Statistik über die Frage der Vererbung bei Tuberkulose zu einem endgültig abschliessenden Urteil zu kommen. Dieses wird wesentlich abhängen ron der Entscheidung der Frage nach der durchschnittlichen Dauer latenter Infektionen bezw. nach der Häufigkeit langdauernder Latenz der Infektion und der damit eng verbundenen Frage nach der Häufigkeit der Infektion in den einzelnen Altersklassen. Über die letztere Frage können einzig allein zahlreiche Sektionen von an Unfall gestorbenen Personen Aufschluss geben, wobei die soziale Stellung und das Alter genau zu unterscheiden wäre. Durch Kombination solcher Ergebnisse mit der bekannten Sterblichkeit an Tuberkulose in den verschiedenen Altersklassen wäre es möglich die Wahrscheinlichkeit der Infektion in den den einzelnen Altersklassen nach den Grundsätzen der Sterbetafeln zu berechnen. Bedenkt man, dass in Berlin jährlich 1000 Personen in Spitälern an Unfall sterben, so sollte ein genügendes Material zu dieser Frage nicht allzuschwer zusammenzubringen sein. Je mehr sich die Wahrscheinlichkeit an Tuberkulose zu erkranken auf die verschiedenen Altersklassen verteilt, um so weniger wahrscheinlich dürfte eine häufige langdauernde Latenz der Tuberkulose sein. Erst wenn hierüber Klarheit geschaffen ist, kann über die Bedeutung der Vererbung endgültig abgeurteilt werden. Vorläufig kann man nur von dem Einfluss einer Belastung sprechen, die jedenfalls zu einem grossen Teile auf Infektion zurückzuführen ist. Für die Versicherungsmedizin ist schon dieses Resultat bedeutsam genug. Mir aber genügte es rorläufig einmal gezeigt zu haben, dass das Problem der familiären Belastung bei Tuberkulose einer statistischen Behandlung fähig ist, dass es insbesondere behandelt werden kann, ohne dass man nach dem Vorschlag von Lorenz, Martius und Schlüter auf ausgedehnte Stammtafeln angewiesen jst, und dass eine Wirkung familiärer Belastung entschieden vorhanden ist, sofern sie nicht durch ungenaue Fragestellung verdeckt wird. Auf eine Erklärung des Wesens der Vererbung bei Tuberkulose kann vorläutig verzichtet werden, so lange der Nachweis eines Bestehens derselben nicht exakt erbracht ist. Und dies muss vorläufig zugegeben werden, während ein Bezweifeln des Vorhandenseins der Wirkung der Infektion besonders mit der ungenauen Fragestellung der R if f el schen Arbeiten unter allen Umständen über das Ziel hinausschiesst. 


\section{Ergebnisse.}

Das Problem der familiären Belastung bei Tuberkulose lässt sich bei geeigneter Fragestellung und mit Ausschluss des Einflusses ron Alter, sozialer Stellung und Beruf statistisch behandeln und ergibt das Vorhandensein einer ätiologischen Bedeutung der Tatsache der Belastung, namentlich von seiten der Mutter, für die Sterblichkeit an Tuberkulose. Der Einfluss der Belastung auf den Verlauf der Tuberkulose kann nicht als genügend untersucht bezeichnet werden. Ob es sich bei dem stärkeren Einfluss der tuberkulösen Mutter um eine Addition von Vererbung und vermehrter Infektionsgelegenlieit oder um letztere allein handelt, kann nicht entschieden werden. Der Einfluss der tub rkulösen Mutter zeigt sich ganz besonders bei der Kindersterblichkeit. Häufig besteht auch bei tuberkulösen Erwachsenen ein Zusammenhang mit frühem Tod der Eltern an Tuberkulose, doch lässt sich nicht der ganze Einfluss der Belastung auf Erwachsene durch vermehrte Infektionsgelegenheit im Kindesalter erklären.

Jedenfalls erreichen die Kinder, welche durch ihre Eltern im Säuglingsalter mit Tuberkulose infiziert zu werden Gelegenheit hatten, nur sehr selten das Alter der Erwachsenen. Weit wichtiger ist das spätere Kindesalter. Die Säuglingsmilch kann nicht die wesentliche Ursache der Tuberkulose Erwachsener sein.

Wenn man den ganzen Einfluss der Belastung auf Infektion zurückführen will, so muss man sowohl eine Infektion in jedem Alter, wie eine häufige lange Latenz der Infektion nebeneinander annehmen. Beides schliesst sich bis zu einem gewissen Grẫ aus. Für die Wirkung der Infektion bei Erwachsenen spricht auch der von mir nachgewiesene Einfluss des Zusammenlebens mit tuberkulösen Ehegatten.

Von der Lösung der Frage nach dem Vorkommen häufiger langdauernder Latenz der Infektion hängt auch die der Möglichkeit hereditärer Einflüsse sehr wesentlich ab.

Numerisch hat die Belastung ungefähr denselben Einfluss, wie das Zusammenleben mit tuberkulösen Ehegatten; darin scheint ein. gewisser Widerspruch mit der ausschliesslichen Erklärung des Einflusses der Belastung durch Infektion zu liegen, ebenso in dem starken Einfluss der Belastung bei günstigen beruflichen und sozialen Verhältnissen der Eltern.

Eine Untersuchung grossen Stils über das Schicksal nachgeborener Kinder tuberkulöser Väter wäre geeignet, die Frage der Vererbung ihrer Lösung näher zu bringen. Dabei müssten Fälle mit und ohne Infektionsgelegenheit durch Mutter und Geschwister unterschieden werden. Die Beschaffung der Grundlagen für eine solche Untersuchung 
liesse sich in Ländern mit geordneter Todesursachenstatistik oder Meldezwang für Todesfälle an Tuberkulose ohne erhebliche Mühe und Kosten durchführen.

\section{Literatur.}

Behring, Tuberkulosebekämpfung. 75. Versammlung deutscher Naturforscher und Ärzte. Cassel 1903.

Boeg, Über erbliche Disposition zur Lungenphthise. Zeitschr. f. Hygiene und Infektionskrankheiten. Bd. L. 1905.

Cornet, Die Tuberkulose. Wien 1900 u. 1906. Siehe dort auch die hier nicht angegebene Literatur.

Geissler, Über den Einfluss der Fruchtbarkeit auf die Säuglingssterblichkeit. 7eitschr. d. K. Sächs. Statist. Bureaus. Bd. 35. 1885.

Gottstein, Die frühzeitige Feststellung des Vorhandenseins eiuer Veranlagung zur Tuberkulose, insbesondere zur Lungentuberkulose. IV. Internat. Kongress für Versicherungsmedizin. Berlin 1906.

Jakob und Pannwitz, Entstehung und Bekämpfung der Lungentuberkulose. Berlin 1901.

Kirchner, Die Gefahren der Eheschliessung von Tuberkulösen and deren Verhütung und Bekämpfung. Kongress für Bekämpfung der Tuberkulose als Volkskrankheit. Berlin 1899.

Kuthy, Klinisch-statistischer Beitrag zur Frage der Verbreitungsweise der Tuberkulose. Pester med. chirurg. Presse. 1894. Nr. 51.

Lorenz, Lehrbuch der gesamten wissenschaftlichen Geneologie. 1898.

Martius, 1. Das Vererbungsproblem in der Pathologie. Berl. klin. Wochenschr. Nr. 30 u. 31.

2. Die Vererbbarkeit der konstitutionellen Faktore der Tuberkulose. Ebenda. 45.

3. Über die Bedentung der Vererbung und Disposition in der Pathologie mit besonderer Berïcksichtigung der Tuberkulose. Kongress f. inn. Medizin. 1905.

Rejche, 1. Die Erfolge der Heilstättenbehandlung Lungenschwindsüchtiger und klinische Bemerkungen zur Tuberculosis pulmonum. Deutsche med. Wochenschr. 1899. Nr. 34.

2. Die Bedeutung der erblichen Belastung bei der Lungenschwindsucht. Zeitschr. f. Tuberkulose u. Heilstättenwesen. Bd. I. 1900.

Riffel, 1. Die Erblichkeit der Schwindsucht und tuberkulösen Prozesse. Karlsruhe 1891.

2. Mitteilungen über die Erblichkeit und Infektiosität der Schwindsucht. Braunschweig 1892.

3. Weitere pathogenetische Studien über Schwindsucht und Krebs. Frankfurt 1901.

4. Schwindsucht und Krebs im Lichte vergleichend geneologischer Forschung. Karlsrube 1905. 
Schlossmann, Die Tuberkulose im frühen Kindesalter. Beiträge zur Klinik der Tuberkulose. Bd. VI. 1906.

Schlüter, Die Anlage zur Tuberkulose. Leipzig 1905.

Schwarzkopf, Bedeutung von Infektion, Heredität und Disposition für die Entstehung der Lungentuberkulose. Deutsch. Archiv f. klin. Med. Bd. 78. 1903.

Turban, Beiträge zur Kenntnis der Iungentuberkulose. Wiesbaden 1899.

W. Weinberg, 1. Pathologische Vererbung und genealogische Statistik. Deutsch. Arch. f. klin. Med. Bd. 78. 1903.

2. Die Tuberkulose in Stuttgart. Med. Korr.-Blatt des Württ. ärztl. Landesvereins. Bd. LXXVI. 1906. Nr. 1 u. 2.

3. Tuberkulose beider Ehegatten. Beiträge zur Klinik der Tuberkulose. Bd. V. 1906.

4. Die Beziehungen zwischen der Tuberkulose und Schwangerschaft. Geburt und Wochenbett. Ebenda. Bd. V. 1906.

5. Tuberkulose und Familienstand. Zentralbl. f. allgem. Gesundheitspflege. Bd. XXV. 1906.

Weismann, Die Kontinuität des Keimplasmas als Grundlage einer Theorie der Vererbung. Jena 1885.

Westergaard, Über die Vererbung der Schwindsucht. Assekuranz-Jahrbuch.

Bd. XIV. 1893. 